\title{
Over-expression of poplar NAC15 gene enhances wood formation in transgenic tobacco
}

\author{
Wenjing Yao ${ }^{1,2}$, Dawei Zhang ${ }^{1}$, Boru Zhou ${ }^{1 *}$, Jianping Wang $^{3}$, Renhua $\mathrm{Li}^{1}$ and Tingbo Jiang ${ }^{1 *}$
}

\begin{abstract}
Background: NAC (NAM/ATAF/CUC) is one of the largest plant-specific transcription factor (TF) families known to play significant roles in wood formation. Acting as master gene regulators, a few NAC genes can activate secondary wall biosynthesis during wood formation in woody plants.

Results: In the present study, firstly, we screened 110 differentially expressed NAC genes in the leaves, stems, and roots of di-haploid Populus simoniixP. nigra by RNA-Seq. Then we identified a nucleus-targeted gene, NAC15 gene, which was one of the highly expressed genes in the stem among 110 NAC family members. Thirdly, we conducted expression pattern analysis of NAC15 gene, and observed NAC15 gene was most highly expressed in the xylem by RT-qPCR. Moreover, we transferred NAC15 gene into tobacco and obtained 12 transgenic lines overexpressing NAC15 gene (TLs). And the relative higher content of hemicellulose, cellulose and lignin was observed in the TLs compared to the control lines containing empty vector $(C L s)$. It also showed darker staining in the culms of the TLs with phloroglucinol staining, compared to the CLs. Furthermore, the relative expression level of a few lignin- and cellulose-related genes was significantly higher in the TLs than that in the CLs.
\end{abstract}

Conclusions: The overall results indicated that NAC15 gene is highly expressed in the xylem of poplar and may be a potential candidate gene playing an important role in wood formation in transgenic tobacco.

Keywords: Populus simoniixP. nigra, NAC, Transcription factor, Lignin, Wood formation

\section{Background}

As one of the most widely and environmentally natural materials, wood is generally used in the construction, paper-making, transportation, chemical industry, military, aerospace and other industries, as well as the production of various wood products, such as agricultural tools, furniture, handicrafts, and musical instruments. Woody biomass can also be utilized as a sustainable and carbon-neutral resource for bioenergy [1]. The demand for wood always increases as it is a cost-effective and renewable resource for industry and energy [2]. There are mainly two kinds of cells with secondary cell walls in the process of wood formation, fibres and tracheary elements. The formation of the two types of cells goes through cell expansion, deposition of secondary walls,

\footnotetext{
*Correspondence: 896099005@qq.com; tbjiang@yahoo.com

'State Key Laboratory of Tree Genetics and Breeding, Northeast Forestry

University, 51 Hexing Road, Harbin 150040, China

Full list of author information is available at the end of the article
}

lignification and programmed cell death (PCD) [3]. Understanding the process of wood formation contributes to wood property and production, which has significant implication in tree biology and biotechnology.

As a model tree, Populus is usually used to understand the unique processes that occur in woody plants, including wood formation $[4,5]$. The molecular and genetic mechanisms regulating wood formation in Populus have been studied by developmental genetic, genomic and biochemical approaches [6]. The identification of expressed sequence tags (ESTs), hormones and genes regulating wood formation is getting popularity in Populus [7-9]. For example, $4 \%$ of 5692 ESTs from two poplars were identified to be involved in various processes of cell wall formation, such as lignin and cellulose synthesis [7]. A unique tissuespecific transcript analysis revealed that lignin and cellulose biosynthetic-related genes, transcription factors (TFs) and other potential regulators of xylogenesis were under strict developmental stage-specific transcriptional regulation in

(c) The Author(s). 2020 Open Access This article is distributed under the terms of the Creative Commons Attribution 4.0 International License (http://creativecommons.org/licenses/by/4.0/), which permits unrestricted use, distribution, and 
poplar [10]. In particular, several TFs such as AUXIN RESPONSE FACTOR (ARF), CLASS III HOMEODOMAINLEUCINE ZIPPER (HD-ZIPIII), KANADI (KAN), MYB and NAC might govern the complex networks of transcriptional regulation in wood formation in poplar $[9,11]$.

NAC family is one of the largest plant-specific TF families known to play significant roles in wood formation [12]. A few NAC genes can activate secondary wall biosynthesis during wood formation acting as master gene regulators, such as vascular-related NAC-domain genes $(V N D)$ and secondary wall-associated NAC domain genes $(S N D)[13,14]$. Transcriptional profiling indicated there were seven $V N D$ genes expressed preferentially in the developing vascular tissues in Arabidopsis [15]. Out of them, VND6 and VND7 are key regulators of xylem vessel differentiation. They regulate the expression of a broad range of genes involved in xylem vessel formation $[16,17]$. Two NAC domain TFs, SND1 and NST1 (NAC secondary wall thickening promoting factor 1) were proved to function redundantly in regulation of secondary wall synthesis in Arabidopsis [18]. Except NAC genes from Arabidopsis, many wood-associated NAC domain (WND) genes from Populus were identified to be master regulators in wood formation. For example, overexpression of two NAC genes from Populus trichocarpa, PtrWND2B and PtrWND6B, leaded to ectopic deposition of cellulose, xylan, and lignin in Arabidopsis by inducing the expression of secondary wallassociated TFs and secondary wall biosynthetic genes [14]. Chimeric repressor of a secondary wall-associated NAC gene from Populus (PtSND2) severely affected wood formation in transgenic $P$. davidiana $\times P$. bolleana by down-regulating a number of wood-associated genes [19].

NAC transcription regulators in wood formation precisely coordinate the expression of secondary wall-related genes, which requires fine temporal and spatial regulation [14, 20]. There were 289 putative NAC genes in Populus trichocarpa, and most of them showed different temporal and spatial expression patterns [21, 22]. In this study, firstly, we screened differentially expressed NAC genes in the leaves, stems, and roots of di-haploid Populus simonii $\times$ P. nigra by RNA-Seq. Then we conducted expression pattern analysis of NAC15 gene in the different tissues by RT-qPCR. Thirdly, we confirmed subcellular localization of NAC15 gene by particle bombardment. Moreover, we transformed the gene into tobacco through Agrobacterium-mediated method and performed physiological, histological and molecular analysis of transgenic tobacco lines overexpressing NAC15 gene. The study indicated NAC15 gene from poplar plays an important role in wood formation in transgenic tobacco.

\section{Results}

Transcriptome analysis of NAC family in Populus simonii $\times P$. nigra

The mRNA abundance of each gene in each sample was profiled as fragment per kilo bases per million reads
(FPKM). The FPKM information of all 289 NAC members in the roots, stems and leaves of Populus simonii $\times$ P. nigra was retrieved from RNA-seq data (Additional file 3: Excel S1). There were a total of 231 NAC genes detected by RNASeq. Based on FPKM $\geq 4$ in at least one tissue, 126 out of the 231 genes were screened to count the expression of NAC genes. Out of the 126 genes, there were 115, 123, 118 differentially expressed genes in the comparison pairs between leaves and stems, roots and stems, leaves and roots, respectively. As many as 110 NAC genes were differentially expressed in the three tissues. The heatmap of the 110 genes showed the expression pattern in the leaves and stems can be clustered together, which indicated the genes have similar expression pattern in the two tissues (Fig. 1).

\section{Phylogenetic analysis of NAC15}

The $1257 \mathrm{bp}$ coding sequence of NAC15 gene from Populus simonii $\times$ P. nigra (Potri.001G448400.1) contains an ORF encoding 418 amino acids. Amino acids sequence blasts indicated that NAC15 from poplar shared 86, 77, 75, 77, 72, 66, 66, 66, 65, 65\% sequence similarity with Salix purpurea (SapurV1A.0131 s0060.3), Ricinus communis (30,068.m002591), Manihot esculenta (Manes.02G001600.1), Theobroma cacao (Thecc1EG 015621t1), Gossypium raimondii (Gorai.004G129200.1), Prunus persica (Prupe.5G131900.1), Malus domestica (MDP0000762302), Fragaria vesca (mrna01881.1-v1.0hybrid), Eucalyptus grandis (Eucgr.E01053.1), and Vitis vinifera (GSVIVT01019670001), respectively. Multiple amino acids alignment showed that above proteins shared a highly conserved domain of 160 amino acids, namely NAC domains, which can be divided into A-E sub-domains (Fig. 2a). The phylogenetic tree with the top 10 identical protein sequences indicated that NAC15 from poplar had relatively high homology with the proteins from willow, cassava and castor-oil plant, while had relatively low homology with those from wildstrawberry, peach tree and apple tree (Fig. 2b).

\section{Localization of NAC15 protein}

As shown in Fig. 3, the fluorescence signal of NAC15GFP (green fluorescent protein) fusion was detected in the nucleus while the control was fully expressed in the cell, which revealed NAC15 protein was localized to the nucleus. To confirm the result, the NAC15-GFPtransfected onion cells were stained with DAPI and observed under immunofluorescence microscope. The combined fluorescence signal of DAPI and GFP was consistently in the nuclei (Additional file 4: Figure S1), which exactly proved nuclear localization of NAC15.

\section{Expression pattern analysis of $\mathrm{NAC} 15$ gene}

NAC15 gene was differentially expressed in the leaves, stems and roots, and its mRNA abundance was the 


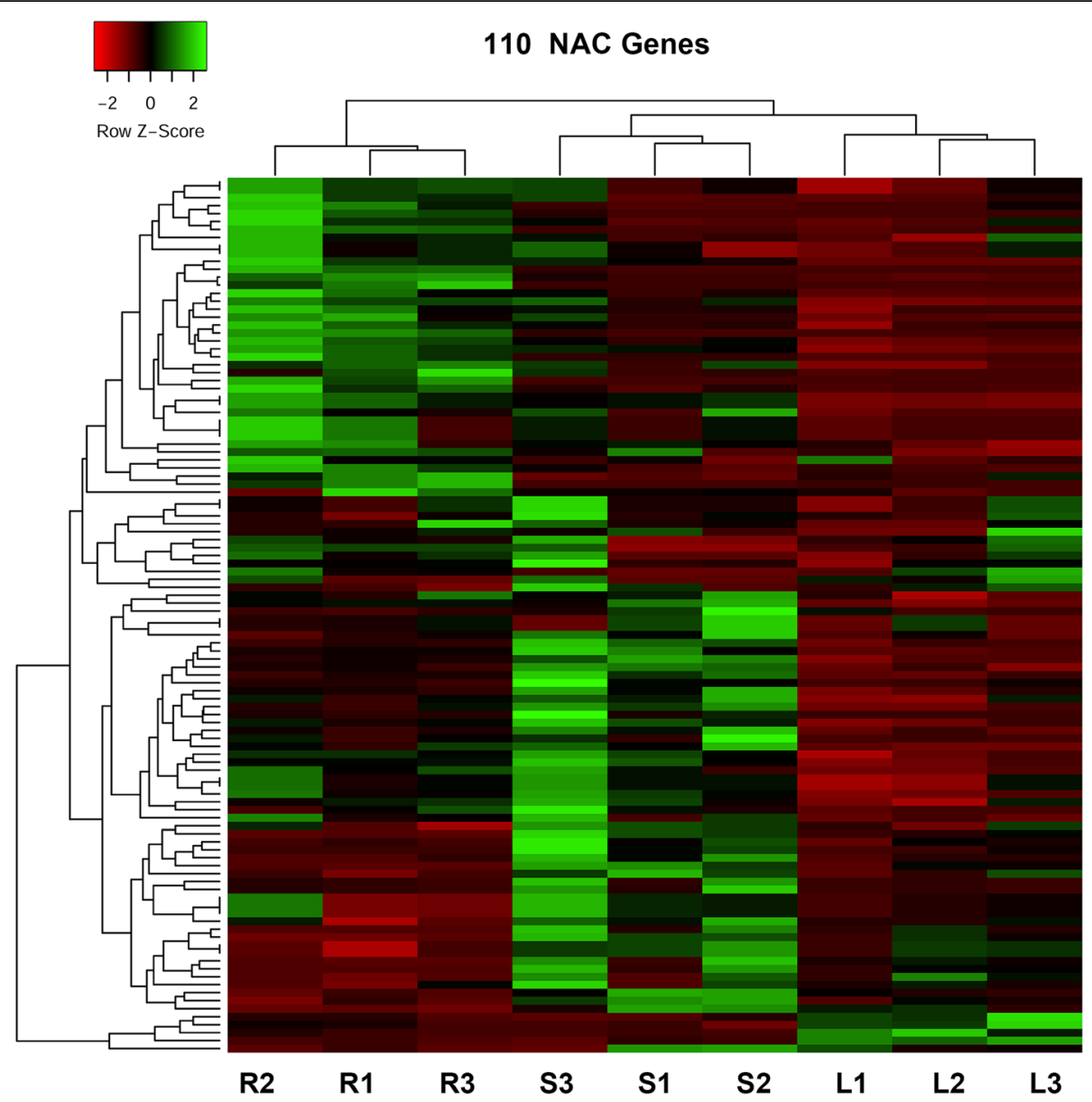

Fig. 1 The heatmap of differentially expressed 110 NAC genes in the three tissues of Populus simoniix P.nigra. The heatmap was drawn by Heatmapper (http://www.heatmapper.ca/expression/). Red and green colors indicate low and high expression, respectively. R1-3, S1-3 and L1-3 indicate roots, stems and leaves with three biological repeats, respectively

highest in the stems, followed by leaves and roots based on RNA-Seq. The relative expression level of NAC15 gene in different tissues at different developmental stage was quantified by RT-qPCR. The results indicated the expression pattern of NAC15 gene was hugely diverse at different tissues and displayed a rapid decrease from $\mathrm{xy}$ lems and leaves to cambiums and roots. The highest expression level appeared in the secondary xylems and the lowest was in the roots, which was in accordance with RNA-Seq results in trend. And the highest expression level was about 173 times higher than the lowest (Fig. 4). The relative expression level of NAC15 gene was also significantly different during developing stages. For example, it was higher in the secondary xylems than that in the primary and crude xylems of poplar (Fig. 4). In conclusion, the expression of NAC15 gene had spatiotemporal specificity, and its expression pattern may play a pivotal role in the temporal and spatial regulation of wood-associated genes in the process of wood formation.

\section{Generation of trangenic tobacco overexpressing NAC15 gene}

We obtained 18 transgenic tobacco lines including 12 TLs and 6 CLs. The transgenic tobacco was confirmed by PCR and RTPCR. As shown in Fig. 5, the expected bands were amplified in the TLs, but not in the CLs and wild type (WT) plants, which proved successful integration of NAC15 gene in tobacco.

\section{Gene expression analysis of lignin- and cellulose-related genes by RT-qPCR}

A few lignin- and cellulose-related genes, such as CesA (Cellulose synthase), $C 4 H$ (Cinnamate 4-hydroxylase), $C A D$ (Cinnamyl alcohol dehydrogenase), $P A L$ (Phe ammonia-lyase), $C L$ (Coumarate: coenzyme A ligase), CCOMT (Caffeoyl-CoA Omethyltransferase) etc. (Additional file 1: Table S1) were required for secondary wall biosynthesis in plants [23, 24]. Taken $\operatorname{Ces} A$ as an example, PtoCesA3 was highly expressed during primary cell wall formation and was proved to be associated with growth and wood properties of Populus 
a

MDP0000762302 (Malus domestica) Prupe.5G131900.1 (Prunus persica) mrna01881.1-v1.0-hybrid (Fragaria vesca) GSVIVT01019670001 (Vitis vinifera) Thecc1EG015621t1 (Theobroma cacao) Manes.02G001600.1 (Manihot esculenta) SapurV1A.0131s0060.3 (Salix purpurea) 30068.m002591 (Ricinus communis) Gorai.004G129200.1 (Gossypium raimondii) Eucgr.E01053.1 (Eucalyptus grandis) Potri.001G448400.1 (Populus trichocarpa) NAC15 (Populus simonii × P. nigra)

MDP0000762302 (Malus domestica) Prupe.5G131900.1 (Prunus persica) mrna01881.1-v1.0-hybrid (Fragaria vesca) GSVIVT01019670001 (Vitis vinifera) Thecc1EG015621t1 (Theobroma cacao) Manes.02G001600.1 (Manihot esculenta) SapurV1A.0131s0060.3 (Salix purpurea) 30068. m002591 (Ricinus communis) Gorai.004G129200.1 (Gossypium raimondii) Eucgr.E01053.1 (Eucalyptus grandis) Potri.001G448400.1 (Populus trichocarpa) NAC15 (Populus simonii $\times$ P. nigra)

MDP0000762302 (Malus domestica) Prupe.5G131900.1 (Prunus persica) mrna01881.1-v1.0-hybrid (Fragaria vesca) GSVIVT01019670001 (Vitis vinifera) Thecc1EG015621t1 (Theobroma cacao) Manes.02G001600.1 (Manihot esculenta) SapurV1A.0131s0060.3 (Salix purpurea) 30068.m002591 (Ricinus communis) Gorai.004G129200.1 (Gossypium raimondii) Eucgr.E01053.1 (Eucalyptus grandis) Potri.001G448400.1 (Populus trichocarpa) NAC15 (Populus simonii $\times$ P. nigra)

\section{b}
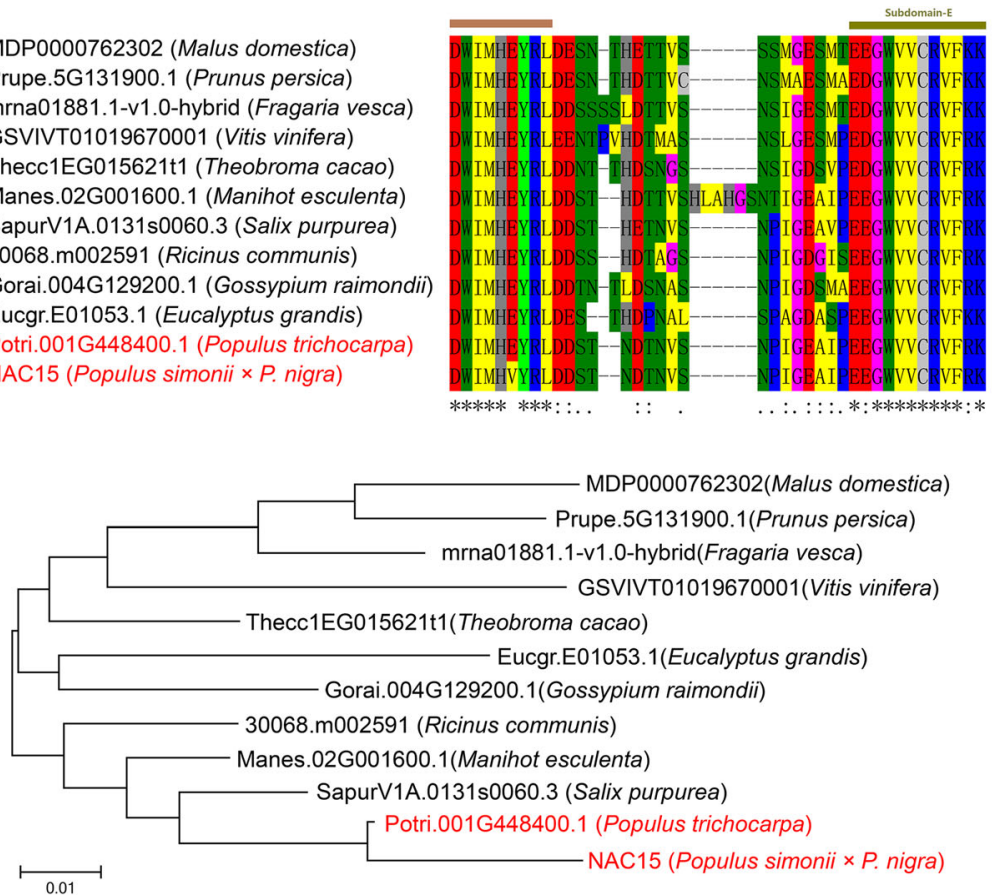
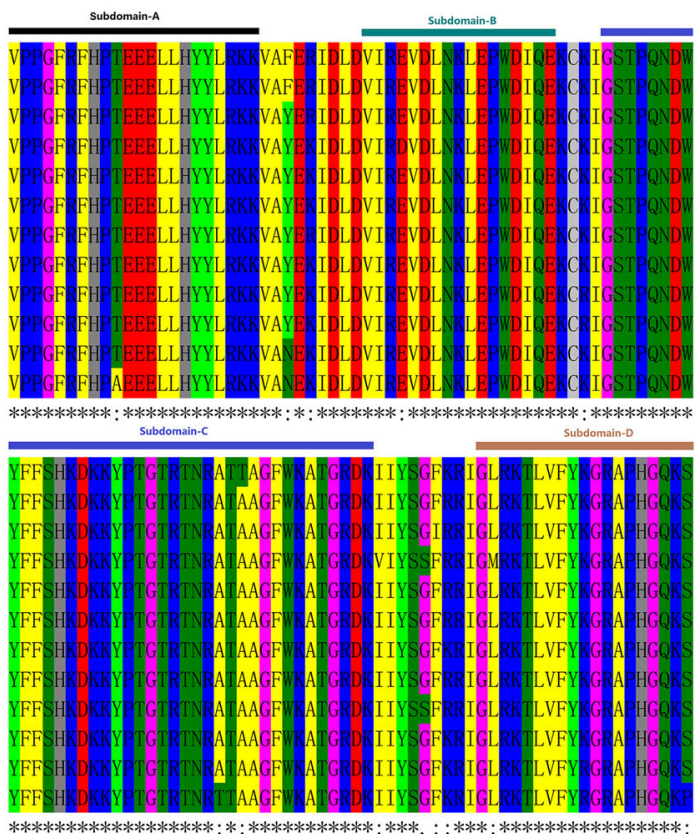

Fig. 2 Conserved domain alignment and phylogenetic analysis of NACs from 12 different plant species. The conserved NAC domain can be divided to 5 sub-domains (A-E). The colorful horizontal bars represent the start and end positions of each sub-domain. a Domain alignment of NACs by Clustal W; b Phylogenetic tree of NACs constructed by Neighbor-Joining method with MEGA 6 program

tomentosa [25]. PAL1 and PAL2 were identified to have relationship with tissue-specific lignin synthesis [26]. RT-qPCR was conducted to detect the relative expression level of ligninand cellulose-related genes in the transgenic plants. The results indicated the relative expression level of $\operatorname{Ces} A, C A D$, $P A L, 4 C L$, and $C 4 H$ etc. in the TLs was significantly higher than that in the CLs (Fig. 6).

\section{Histological analysis of transgenic tobacco overexpressing NAC15 gene}

There are three types of polymers (hemicelluloses, cellulose and lignin) in the secondary cell wall of plants [23, 27]. Cellulose is the most abundant polysaccharide in plants and its microfibrils can form a main load-bearing network. Hemicellulose mainly consists of xylans, glucans, and mannans. 


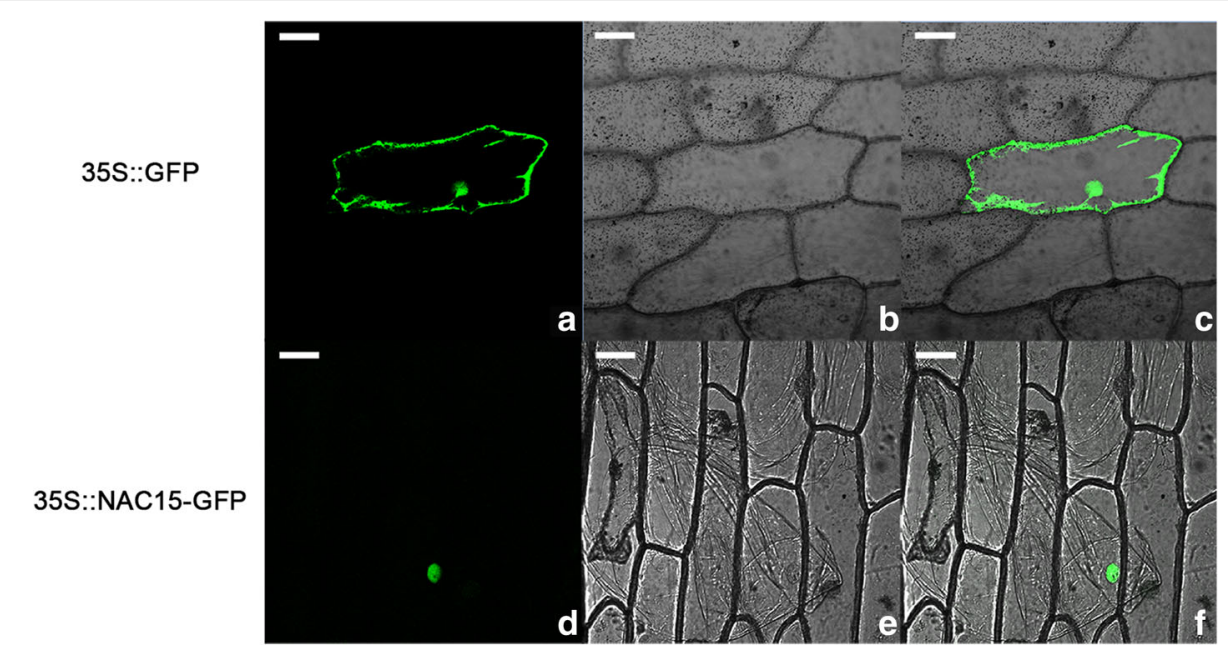

Fig. 3 Subcellular localization of NAC15 in onion epidermal cells by particle bombardment. NAC15 was localized to the nucleus. a-c The GFP fluorescence signals of 35S::GFP vector; $\mathbf{d}$-f The GFP fluorescence signals of 35 s::NAC15-GFP fusion construct. $\mathbf{a}$ and $\mathbf{d}$, dark field; $\mathbf{b}$ and $\mathbf{e}$, bright field; $\mathbf{c}$ and $\mathbf{f}$, overlay of dark field and bright field. Scale bar $=20 \mu \mathrm{m}$

Lignin affects 'waterproofing' capacity, mechanical strength, rigidity and environmental protection of plants [23, 27]. The relative content of hemicellulose, cellulose and lignin was determined to compare wood properties between TLs and CLs. The results showed that the relative content of hemicellulose, cellulose and lignin in the TLs was 1.09$1.38,1.29-1.40,1.31-1.58$ times higher than that in the CLs, respectively (Fig. 7).

\section{Phloroglucinol-HCl staining}

Phloroglucinol- $\mathrm{HCl}$ staining method is commonly used for the characterization of plant lignifications [28]. Therefore, phloroglucinol- $\mathrm{HCl}$ staining was conducted to compare wood properties between TLs and CLs in the study. The result showed there was darker staining in the vascular bundles of TLs, compared to the CLs (Fig. 8). It showed three levels of stem lignifications based on the staining color in the TL1, TL3 and TL5, which was in accordance with the relative content of lignin and relative expression level of ligninrelated genes.

\section{Discussion}

NAC genes are important plant-specific TFs, which regulate multiple biological processes such as plant developmental process, metabolism process, abiotic stress and defense response $[22,29]$. The function of NAC genes in wood formation has come under the spotlight. NAC genes are expressed preferentially in developing wood and differentiating tracheary elements $[11,15]$. In particular, $V N D /$ $N S T / S N D$ subfamilies of NAC domain proteins participate

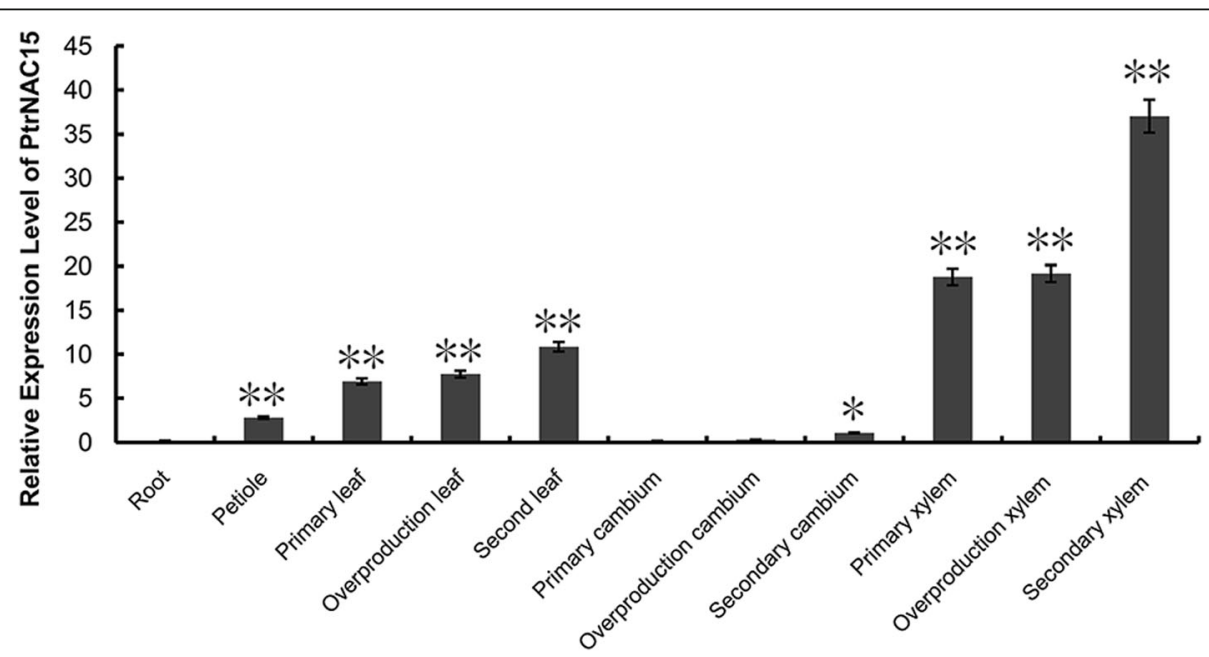

Fig. 4 Expression pattern analysis of NAC15 gene. NAC15 gene was most highly expressed in the xylem. Mean values and standard errors were calculated from three technical replicates by $2^{-\Delta \Delta C t}$ method with three independent biological replicates. ${ }^{*}$ indicates $P<0.05,{ }^{* *}$ indicates $P<0.01$ 


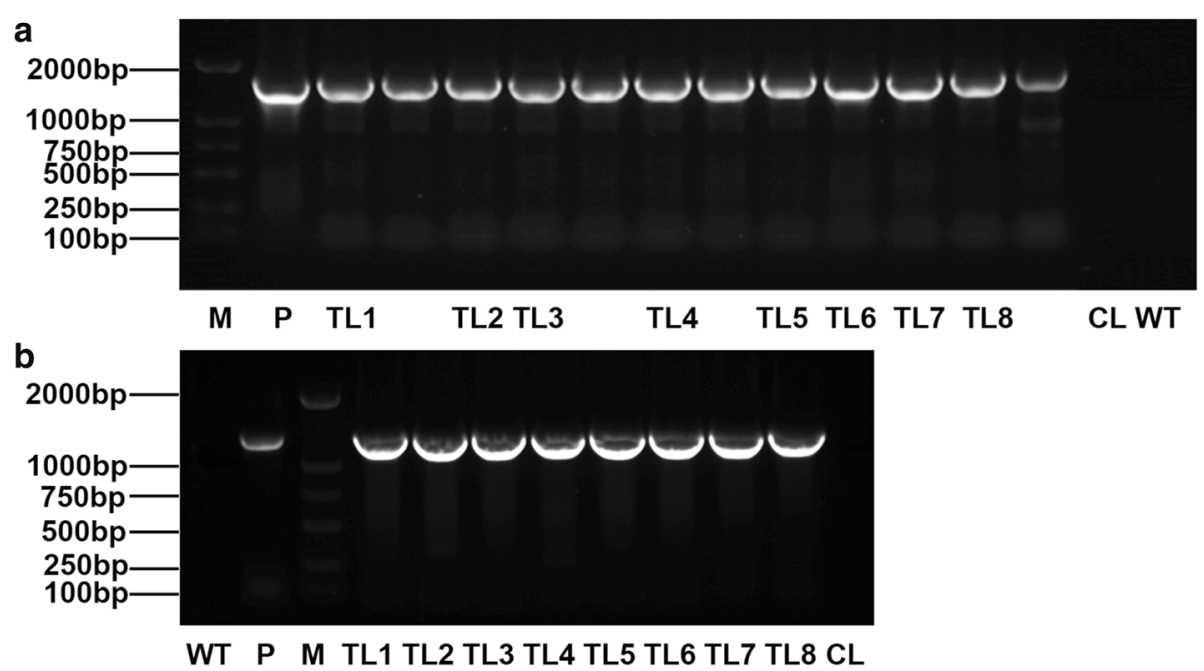

Fig. 5 PCR identification of transgenic tobacco lines. a PCR detection of the transgenic lines with DNA as template; $\mathbf{b}$ RT-PCR detection of the transgenic lines with cDNA as template. M, DL2000 marker; P, positive control use pBl121-NAC15 vector as template; TL1-8, transgenic lines; $C L$, control line; WT, wild type

in transcriptional control of secondary cell wall formation as master switch [11, 12, 30] (Additional file 5: Figure S2). $V N D$ proteins control the expression of genes involved in both secondary wall formation and PCD while NST genes play pivotal roles in transcriptional regulation of secondary wall formation [11, 16, 31] (Additional file 5: Figure $\mathrm{S} 2$ ). VND and NST genes with their downstream genes including other NAC domain proteins, MYB proteins, and homeobox proteins form a transcriptional network regulating secondary wall formation during wood formation $[9,30,31]$ (Additional file 5: Figure S2).
Considerable effort has been made to shed light on the NAC genes in the wood formation of woody plants. It was proposed the existed reciprocal cross-regulation of $V N D$ and SND multi-gene TF families maintain homeostasis in xylem differentiation in Populus trichocarpa [32]. Prominently, wood-associated NAC master switches from poplar (PtrWNDs) are preferentially expressed in the developing wood and key regulators of the biosynthesis of cellulose, xylan, and lignin [14] (Additional file 5: Figure S2). Overexpression of PtrWNDs led to ectopic deposition of wood components in transgenic poplar [1, 20]. Additionally,

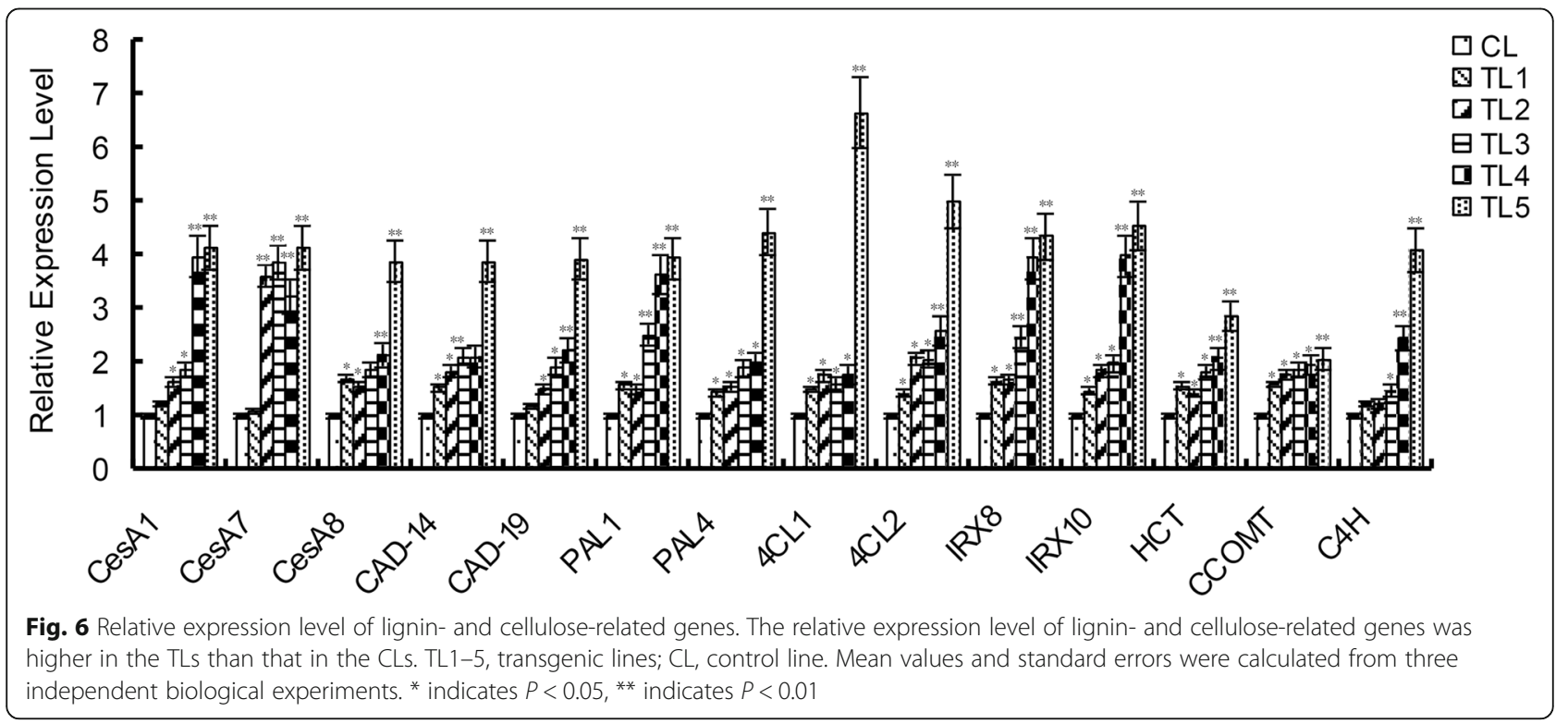




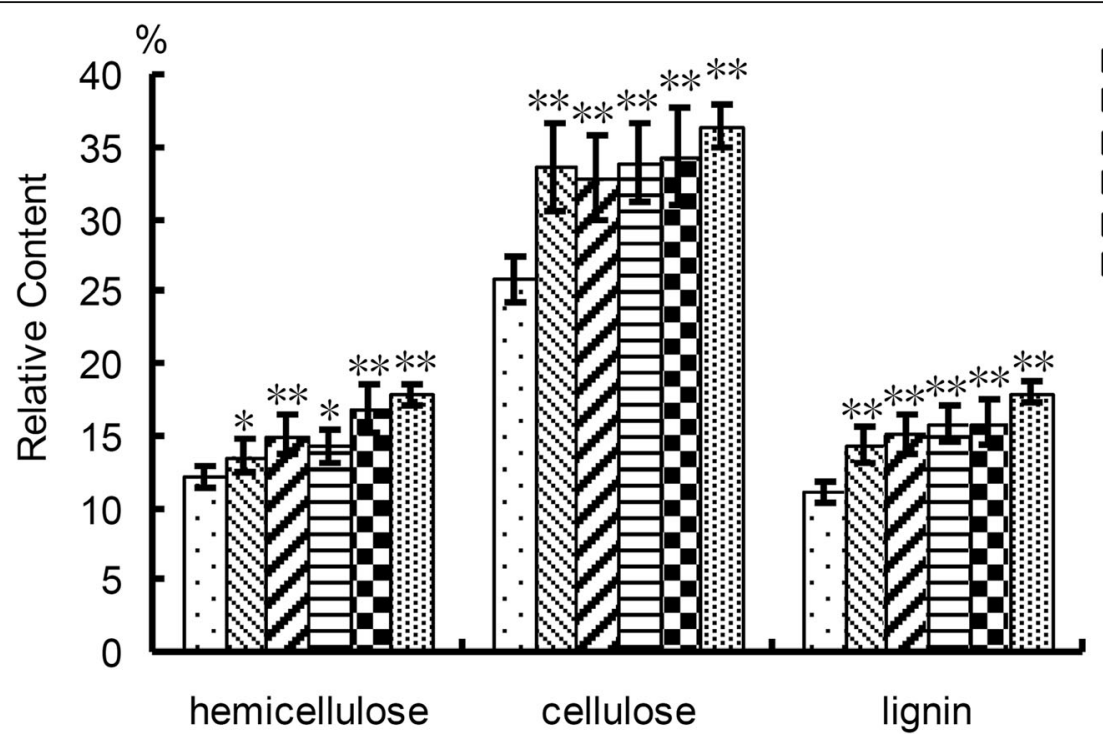

Fig. 7 Wood property comparison of tobacco plants. The relative content of hemicellulose, cellulose and lignin was higher in the TLs than that in the CLs. TL1-5, transgenic lines; $C L$, control line. Mean values and standard errors were calculated from three independent biological experiments. * indicates $P<0.05$, ${ }^{* *}$ indicates $P<0.01$

dominant repression of PtrWNDs caused a drastic reduction of secondary wall thickening in transgenic poplar [13, 20]. PtrWNDs can regulate a suite of downstream woodassociated TFs and wood biosynthetic genes to activate entire secondary wall biosynthetic program in Populus trichocarpa [14, 20] (Additional file 5: Figure S2). In the study, we identified a nucleus-targeted gene from Populus simonii $\times P$. nigra, NAC15 gene. It was one of the highly expressed NACs in the stem based on RNA-Seq. And expression pattern analysis indicated NAC15 gene was most highly expressed in the xylem. The relative content of hemicellulose, cellulose and lignin was higher in the TLs than that in the CLs. Phloroglucinol staining showed darker staining in the phloem and xylem of the TLs, compared to the CLs. And the relative expression level of a few lignin- and cellulose-related genes was significantly higher in the TLs than that in the CLs. All the results indicated NAC15 gene acting as a member of PtrWNDs plays a significant role in wood formation in transgenic tobacco.

It was well known that many genes with high protein sequence similarity can be clustered into same sub-group and generally possess similar function. Based on phylogenetic analysis of well-known Arabidopsis NAC TFs regulating differentiation of xylem vessels and fiber cells, 16 poplar NAC domain homologs were isolated from Populus trichocarpa. Among them, 12 were identified to be PtrWND genes [1]. NAC15 gene was classed into subgroup (V) with SND1 gene from Arabidopsis, which has been demonstrated as a key transcriptional switch regulating secondary wall synthesis in fibers [33, 34]. This subgroup also contains other wood-associated genes, such as NST1 gene and NST2 gene, which regulate secondary wall thickenings in Arabidopsis [35]. In addition, NAC15 has high homology with WND1A gene, which was identified to regulate cell wall thickening during fiber development in Populus species $[14,36]$. All above also indicated NAC15 gene is associated with wood formation in plants.

\section{Conclusions}

Among 289 NAC family members from Populus simonii $\times$ P. nigra, a total of $115,123,118$ genes were differentially expressed in the comparison pairs between leaves and stems, roots and stems, leaves and roots, respectively. As many as 110 NAC genes were identified to be differentially expressed in the three tissues. Out of them, NAC15 gene was highly expressed in the stem. And the gene was confirmed to be nucleus-targeted. The TLs displayed higher content of hemicellulose, cellulose and lignin, compared to the CLs. Phloroglucinol staining also showed an increase of lignification in the vascular bundles of the TLs, compared to the CLs. The relative expression level of a few lignin- and cellulose-related genes such as CesA, CAD, PAL, $4 C L$, and $C 4 H$ etc. was significantly higher in the TLs than that in the CLs. All the results indicated NAC15 gene from poplar plays an important role in wood formation in transgenic tobacco.

\section{Methods}

\section{Plant materials and culture}

Populus simonii $\times$ P. nigra is a specific hybrid poplar widely grown in the northeast, northwest and southwest of China. The growing twigs of wild-type Populus simonii $\times P$. nigra from one clone of experimental forest of Northeast Forestry University were hydroponic cultured 


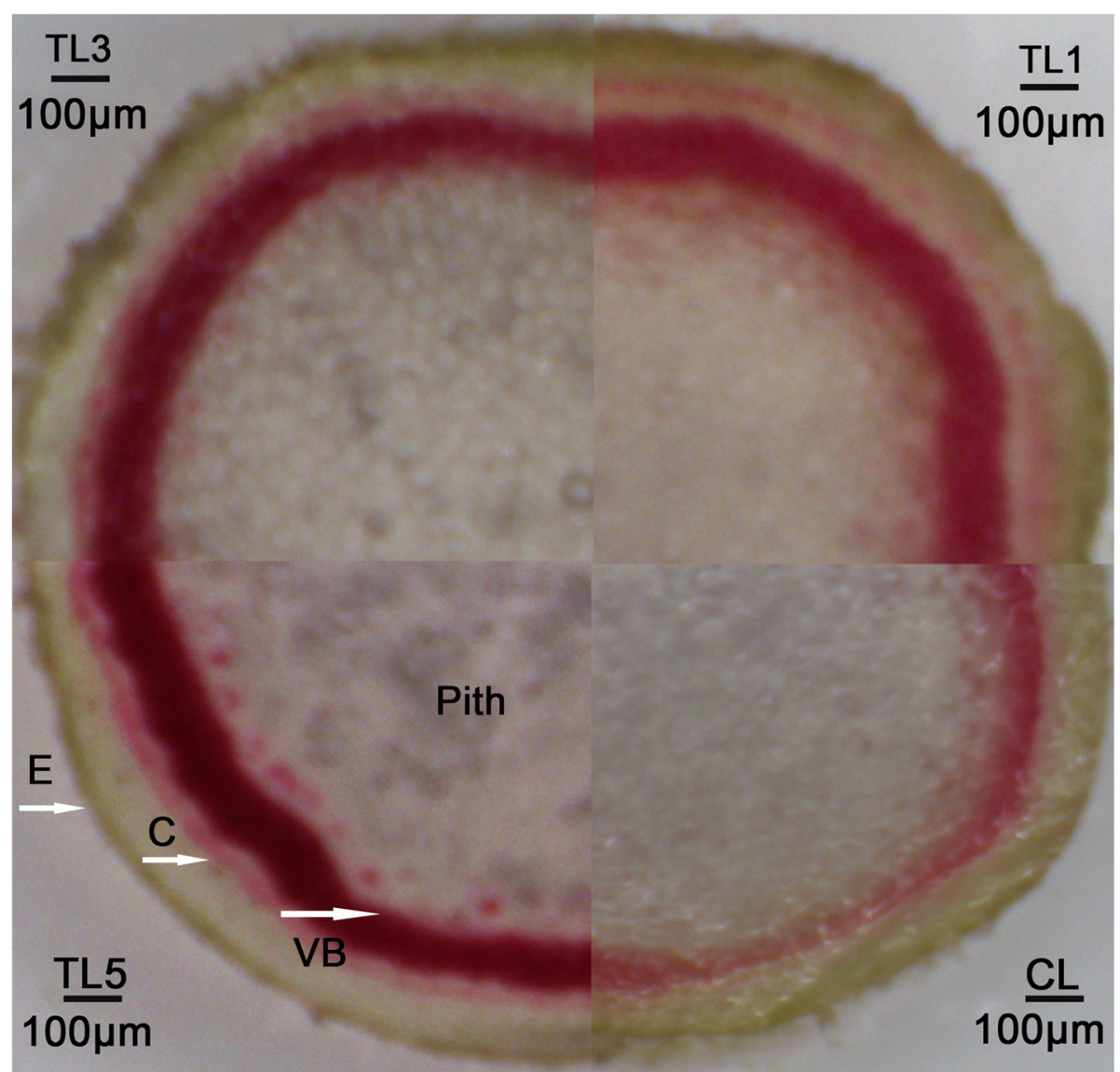

Fig. 8 Phloroglucinol staining of tobacco plants. The staining color was obviously deeper in the TLs than that in the CL. And the three TLs showed three levels of staining color, which indicates three levels of lignification. TL1, 3, 5, transgenic lines; $C L$, control line; $E$, epidermis; $C$, cortex; VB, vascular bundles

at room temperature with $16 / 8$-h light/dark cycles and $70 \%$ relative humidity for two months. The new roots, stems and leaves from the twigs were frozen in liquid nitrogen for RNA-Seq. And the roots, petioles, leaves, xylem and cambiums were harvested for expression pattern analysis. Three biological replicates were prepared for each tissue.

The seeds of wild-type Nicotiana tabacum were originated from state key laboratory of tree genetics and breeding of Northeast Forestry University. To prepare sterilized tobacco explants, the tobacco seeds were sterilized using $70 \%(\mathrm{v} / \mathrm{v})$ ethanol for $30 \mathrm{~s}$, followed by $\mathrm{NaClO}$ solution (1\% $\mathrm{NaClO}, 0.05 \%$ TWEEN20) for $10 \mathrm{~min}$ and rinsed using sterile water for 5 times. Then the seeds were placed on 1/2 MS solid medium ( $\mathrm{pH} 5.8-6.0)$ at $24 \pm 2{ }^{\circ} \mathrm{C}, 16 / 8$-h light/dark cycles for germination. And the germinated seeds were transferred into tissue culture bottles containing 1/2 MS solid medium. The one month old disease free seedlings were used for gene transformation [37].

\section{NAC expression analysis by RNA-Seq}

A total of nine samples including leaves, stems, and roots with respective three biological replicates were shipped with dry ice to GENEWIZ Company (www.genewiz.com) for RNA isolation, mRNA-purification, and RNA-Seq with Illumina Hi-seq platform. The raw sequences were cleaned using Trimmomatic v0.30 [38]. The cleaned reads were aligned to Populus trichocarpa reference genome using STAR 2.4.2a [39]. The mRNA abundance of each gene in each sample was quantified as FPKM.

The FPKM information of 289 NAC family members was drawn from RNA-Seq data (Additional file 3: Excel S1). The NACs with FPKM $\geq 4$ in at least one tissue were applied to count differentially expressed NAC genes in the three tissues. The fold change (FC) in the different tissues was standardized by $\log _{2}$ FPKM ratios [40, 41]. The hierarchical clustering of the differentially expressed NAC genes in the three tissues was conducted by Heatmapper (http://www.heatmapper.ca/expression/). 


\section{RT-qPCR analysis}

The total RNA was extracted using Column Plant RNAout Kit (CAT\#:71203, Tiandz, Beijing, China) and reversetranscribed into cDNA using PrimeScript ${ }^{\mathrm{tw}} \mathrm{RT}$ reagent Kit with gDNA Eraser (RR047A, Takara, Dalian, China). RTqPCR experiment was performed by ABI7500 fast realtime PCR detection system using SYBR Premix Ex Taq"II (DRR081A, TaKaRa, Dalian, China). The relative expression level of genes was calculated by $2^{-\Delta \Delta C t}$ method with three biological replicates [42]. The primer pairs of poplar NAC15 gene (NAC15-1), reference gene, and lignin- and cellulose-related genes (Additional file 2: Table S2) were designed based on Populus trichocarpa v3.1 in Phytozome12 (https://phytozome.jgi.doe.gov/pz/portal.html).

\section{Phylogenetic analysis of NAC15 protein}

Amino acid sequences of NACs from Populus trichocarpa and other species were derived from PlantTFDB (http:// planttfdb.cbi.pku.edu.cn/). Multiple alignment of conserved NAC domain was performed by Clustal W [43]. Phylogenetic tree of NAC proteins was constructed by Neighbor-Joining method with MEGA 6 program [44].

\section{Subcellular localization of NAC15}

The coding region of NAC15 gene without stop codon was cloned into pBI121 vector with specific primers (NAC15-2, Additional file 2: Table S2) and expressed with GFP under the control of CaMV35S promoter. The combined vectors $35 \mathrm{~S}:: \mathrm{NAC} 15-\mathrm{GFP}$ and $35 \mathrm{~S}:: \mathrm{GFP}$ as control were transferred into onion epidermal cells by particle bombardment, separately. The fluorescence signal of GFP and DAPI was detected by fluorescence microscopy system (LSM 700, Zeiss, Germany).

\section{Generation of transgenic tobacco overexpressing NAC15 gene}

The $1515 \mathrm{bp}$ transcript sequence of NAC15 gene was cloned into $\mathrm{pBI} 121$ vector under the control of CaMV35S promoter with specific primers (NAC15-3, Additional file 2: Table S2). The recombined vector and empty vector as control were transformed into EHA105 Agrobacterium strain by electroporation, separately. The transformed EHA105 strain was confirmed by PCR and sequencing.

The tobacco transformation was conducted as following: 1) the leaves from disease free plants at one month old were cut into $1 \mathrm{~cm} \times 1 \mathrm{~cm}$ discs and soaked in the positively transformed EHA105 liquid medium (OD 0.3-0.5) for $10 \mathrm{~min}$; 2) the leave discs were dried with sterilized filter paper and put on 1/2 MS solid medium for co-culture in dark for two days; 3 ) the leave discs were transferred on the pre-cultural medium (1/2 MS solid medium containing $0.5 \mathrm{mg} / \mathrm{L}$ 6-BA, $0.05 \mathrm{mg} / \mathrm{L} \mathrm{NAA}$ and $100 \mathrm{mg} / \mathrm{L} \mathrm{Kan)}$ until callus emerged; 4) healthy callus were transferred on the shooting medium (1/2 MS solid medium containing
$0.1 \mathrm{mg} / \mathrm{L}$ 6-BA, $0.05 \mathrm{mg} / \mathrm{L}$ NAA and $100 \mathrm{mg} / \mathrm{L}$ Kan) until shoots grew; 5) the shoots were transferred into the rooting medium ( $1 / 2 \mathrm{MS}$ containing $0.2 \mathrm{mg} / \mathrm{L}$ IBA and 100 $\mathrm{mg} / \mathrm{L}$ Kan) until roots generated; 6) the transgenic tobacco seedlings were confirmed by PCR and RT-PCR [45]. The specific primer pairs (NAC15-4) for PCR and RT-PCR were list in the Additional file 2: Table S2.

\section{Determination of secondary wall composition}

The relative content of lignin, hemicellulose and cellulose in tobacco plants was measured at maturation stage with three biological replicates. The sample preparations, determination procedures and calculation formulas referred to the description by Sukjun et al. [46].

\section{Histological analysis}

Histological staining was conducted in the tobacco at growth period with three biological replicates. The procedure was as follows: 1) fixed the stems in the FAA solution (70\% ethanol: glacial acetic acid: formaldehyde; $90: 5: 5, \mathrm{v} / \mathrm{v}$ ) and embedded them in the frozen sectioning medium (OCT; Thermo Scientific, Waltham, MA); 2) cut the embedded stems into slices and put the slices on the slides; 3 ) stained the slides with phloroglucinol solution for $2 \mathrm{~min} ; 4$ ) soaked the slides in $50 \%(\mathrm{v} / \mathrm{v}) \mathrm{HCl} ; 5)$ put coverslips on the slides and wiped the slides with lens paper; 6) examined the slides with optical light microscope [47].

\section{Statistics analysis}

All the data in the study were the mean and standard error of three biological replicates. Student's t-test was used to identify significant differences between TLs and CLs. And the statistical significance was controlled at $p<0.05$.

\section{Supplementary information}

Supplementary information accompanies this paper at https://doi.org/10. 1186/s12870-019-2191-2.

\footnotetext{
Additional file 1: Table S1. List of lignin- and cellulose-related genes in tobacco.

Additional file 2: Table S2. List of primer pairs.

Additional file 3: Excel S1. The FPKM information of differentially expressed NAC genes in the roots, stems and leaves of Populus simoniixP. nigra. (XLS $53 \mathrm{~kb}$ )

Additional file 4: Figure S1. Nuclear localization of NAC15 with DAPI staining. A, dark field for DAPl; B, dark field for GFP; C, bright field; D, overlay of DAPI and GFP. Scale bar $=20 \mu \mathrm{m}$.

Additional file 5: Figure S2. Working model of NAC family TFs and their downstream genes in wood formation.

\section{Abbreviations \\ C4H: Cinnamate 4-hydroxylase; CAD: Cinnamyl alcohol dehydrogenase; CCOMT: Caffeoyl-CoA O-methyltransferase; CesA: Cellulose synthase; $\mathrm{CL}$ : Control line containing empty vector; CL: Coumarate: coenzyme A ligase; ESTs: Expressed sequence tags; FPKM: Fragment per kilo bases per million reads; GFP: Green fluorescent protein; NST: NAC secondary wall thickening; PAL: Phe ammonia-lyase; PCD: Programmed cell death; PtrWNDs: Wood-}


associated NAC master switches from poplar; SND: Secondary wall-associated NAC domain; TF: Transcription factor; TL: Transgenic line overexpressing NAC15 gen; VND: Vascular-related NAC domain; WND: Wood-associated NAC domain; WT: Wild type

\section{Acknowledgements}

Not applicable.

\section{Authors' contributions}

TJ and BZ designed the research. WY conducted experiments, analyzed data and wrote the manuscript. DZ conducted experiments. JW revised the manuscript. RL contributed new analytical tools. All authors read and approved the final manuscript.

\section{Funding}

This work was supported financially by Fundamental Research Funds for the Central Universities (2572018BW04), Natural Science Foundation of Jiangsu Province (BK20190748), and the 111 Project (B16010). The funding bodies were not involved in the study design, data collection, analysis, or preparation of the manuscript.

\section{Availability of data and materials}

All data generated or analysed during this study are included in this published article and its supplementary information files.

\section{Ethics approval and consent to participate}

Not applicable.

\section{Consent for publication}

Not applicable.

\section{Competing interests}

The authors declare that they have no competing interests.

\section{Author details}

${ }^{1}$ State Key Laboratory of Tree Genetics and Breeding, Northeast Forestry University, 51 Hexing Road, Harbin 150040, China. ${ }^{2}$ Co-Innovation Center for Sustainable Forestry in Southern China/Bamboo Research Institute, Nanjing Forestry University, 159 Longpan Road, Nanjing 210037, China. ${ }^{3}$ Department of Agronomy, University of Florida, 2033 Mowry Road, Gainesville, FL 32610, USA

Received: 6 August 2019 Accepted: 8 December 2019

Published online: 08 January 2020

\section{References}

1. Ohtani M, Nishikubo N, Xu B, Yamaguchi M, Mitsuda N, Goué N, Shi F, Ohme-Takagi M, Demura T. A NAC domain protein family contributing to the regulation of wood formation in poplar. Plant J. 2011;67:499-512.

2. Han $\mathrm{KH}, \mathrm{Ko} \mathrm{JH}$, Yang SH. Optimizing lignocellulosic feedstock for improved biofuel productivity and processing. Biofuels Bioprod Biorefin. 2007;1:135-46.

3. Plomion C, Leprovost G, Stokes A. Wood formation in trees. Plant Physiol. 2001;127(4):1513-23.

4. Taylor G. Populus: Arabidopsis for forestry. Do we need a model tree? Ann Bot. 2002;90(6):681-9.

5. Jansson S, Douglas CJ. Populus:a model system for plant biology. Annu Rev Plant Biol. 2007:58(1):435-58

6. Groover AT, Nieminen K, Helariutta Y, Mansfield SD. Wood formation in Populus. In: Genetics and genomics of Populus. New York: Springer; 2010. p. 201-24.

7. Sterky F, Regan S, Karlsson J, et al. Gene discovery in the wood-forming tissues of poplar: analysis of 5,692 expressed sequence tags. Proc Natl Acad Sci. 1998;95(22):13330-5.

8. Sorce C, Giovannelli A, Sebastiani L, Anfodillo T. Hormonal signals involved in the regulation of cambial activity, xylogenesis and vessel patterning in trees. Plant Cell Rep. 2013;32(6):885-98.

9. Ye ZH, Zhong R. Molecular control of wood formation in trees. J Exp Bot. 2015;66(14):4119-31

10. Hertzberg M, Aspeborg $\mathrm{H}$, Schrader J, et al. A transcriptional roadmap to wood formation. Proc Natl Acad Sci. 2001;98(25):14732-7.

11. Demura T, Fukuda H. Transcriptional regulation in wood formation. Trends Plant Sci. 2007;12(2):64-70.
12. Wang $H Z$, Zhao $Q$, Chen F, Wang MY, Dixon RA. NAC domain function and transcriptional control of a secondary cell wall master switch. Plant J. 2011; 68:1104-14.

13. Grant EH, Fujino T, Beers EP, Brunner AM. Characterization of NAC domain transcription factors implicated in control of vascular cell differentiation in Arabidopsis and Populus. Planta. 2010;232:337-52.

14. Zhong $\mathrm{RQ}$, Lee $\mathrm{CH}$, Ye ZH. Functional characterization of poplar woodassociated NAC domain transcription factors. Plant Physiol. 2010;152:1044-55.

15. Kubo M, Udagawa M, Nishikubo N, et al. Transcription switches for protoxylem and metaxylem vessel formation. Genes Dev. 2005;19:1855-60.

16. Yamaguchi M, Goué N, Igarashi H, Ohtani M, Nakano Y, Mortimer JC, et al. Vascular-related NAC-domain 6 (VND6) and VND7 effectively induce transdifferentiation into xylem vessel elements under control of an induction system. Plant Physiol. 2010;153:906-14.

17. Yamaguchi M, Mitsuda N, Ohtani M, Ohme-Takagi M, Kato K, Demura T. Vascular-related NAC-domain 7 directly regulates the expression of a broad range of genes for xylem vessel formation. Plant J. 2011;66:12.

18. Zhong R, Richardson EA, Ye ZH. Two NAC domain transcription factors, SND1 and NST1, function redundantly in regulation of secondary wall synthesis in fibers of Arabidopsis. Planta. 2007;225:1603-11.

19. Wang HH, Tang RJ, Liu H, et al. Chimeric repressor of PtSND2 severely affects wood formation in transgenic Populus. Tree Physiol. 2013;33(8):878-86.

20. Zhong RQ, Mccarthy RL, Lee $\mathrm{CH}$, Ye ZH. Dissection of the transcriptional program regulating secondary wall biosynthesis during wood formation in poplar. Plant Physiol. 2011;157:1452-68.

21. Jin J, Tian F, Yang DC, Meng YQ, Kong L, Luo JC, Gao G. PlantTFDB 4.0: toward a central hub for transcription factors and regulatory interactions in plants. Nucleic Acids Res. 2017:45:D1040-5.

22. Hu R, Qi G, Kong Y, Kong D, Gao Q, Zhou G. Comprehensive analysis of NAC domain transcription factor gene family in Populus trichocarpa. BMC Plant Biol. 2010;10(1):145

23. Taylor-Teeples M, Lin L, De Lucas M, Turco G, Toal TW, Gaudinier A, et al. An Arabidopsis gene regulatory network for secondary cell wall synthesis. Nature. 2014;517:571-5.

24. Boerjan W, Ralph J, Baucher M. Lignin biosynthesis. Annu Rev Plant Biol. 2003:54:519-46.

25. Xu BH, Tian JX, Du QZ, Gong CR, Pan W, Zhang DQ. Single nucleotide polymorphisms in a cellulose synthase gene (PtoCesA3) are associated with growth and wood properties in Populus tomentosa. Planta. 2014;240:1269-86.

26. Olsen KM, Lea US, Slimestad R, Verheul M, Lillo C. Differential expression of four Arabidopsis PAL genes; PAL1 and PAL2 have functional specialization in abiotic environmental-triggered flavonoid synthesis. J Plant Physiol. 2008;165:1491-9.

27. Yang $H$, Yan $\mathrm{R}$, Chen $\mathrm{H}$, Lee DH, Zheng C. Characteristics of hemicellulose, cellulose and lignin pyrolysis. Fuel. 2007:86:1781-8.

28. Guo $H$, Wang $Y$, Wang $L$, Hu $P$, Wang $Y$, Jia $Y$, et al. Expression of the MYB transcription factor gene BpIMYB46 affects abiotic stress tolerance and secondary cell wall deposition in Betula platyphylla. Plant Biotechnol J. 2017;15:107-21.

29. Olsen AN, Ernst HA, Leggio LL, Skiver K. DNA-binding specificity and molecular functions of NAC transcription factors. Plant Sci. 2005;169:785-97.

30. Yamaguchi $M$, Demura T. Transcriptional regulation of secondary wall formation controlled by NAC domain proteins. Plant Biotechnol. 2010;27(3):237-42.

31. Zhang J, Nieminen K, Serra JAA, et al. The formation of wood and its control. Curr Opin Plant Biol. 2014:17:56-63.

32. Lin YC, Chen H, Li Q, et al. Reciprocal cross-regulation of VND and SND multigene TF families for wood formation in Populus trichocarpa. Proc Natl Acad Sci. 2017;114(45):E9722-9.

33. Yao WJ, Zhao K, Cheng ZH, Li XY, Zhou BR, Jiang TB. Transcriptome analysis of poplar under salt stress and over-expression of poplar transcription factor NAC57 gene confers salt tolerance in transgenic Arabidopsis. Front Plant Sci. 2018;9:1121.

34. Zhong R, Demura T, Ye ZH. SND1, a NAC domain transcription factor, is a key regulator of secondary wall synthesis in fibers of Arabidopsis. Plant Cell. 2006:18:3158-70.

35. Mitsuda N, Seki M, Shinozaki K, Ohme-Takagi M. The NAC transcription factors NST1 and NST2 of Arabidopsis regulate secondary wall thickenings and are required for anther dehiscence. Plant Cell. 2005;17:2993-3006.

36. Zhao Y, Sun J, Xu P, Zhang R, Li L. Intron-mediated alternative splicing of wood-associated NAC transcription factor $1 \mathrm{~B}$ regulates cell wall thickening during fiber development in Populus species. Plant Physiol. 2014;164:765-76.

37. Yao WJ, Wang L, Zhou BR, Wang SJ, Li RH, Jiang TB. Over-expression of poplar transcription factor ERF76, gene confers salt tolerance in transgenic tobacco. J Plant Physiol. 2016;198:23. 
38. Bolger AM, Lohse M, Usadel B. Trimmomatic: a flexible trimmer for Illumina sequence data. Bioinformatics. 2014;30:2114-20.

39. Dobin A, Davis CA, Schlesinger F, Drenkow J, Zaleski C, Jha S, et al. STAR: ultrafast universal RNA-seq aligner. Bioinformatics. 2013;29:15-21.

40. Yao WJ, Zhang XM, Zhao K, Zhou BR, Li RH, Jiang TB. Expression pattern of ERF gene family under multiple abiotic stresses in Populus simonii $\times$ P. nigra. Frontiers in. Plant Sci. 2017;8:181.

41. Yao WJ, Zhou BR, Zhang XM, Zhao K, Cheng ZH, Jiang TB. Transcriptome analysis of transcription factor genes under multiple abiotic stresses in Populus simonii × P.nigra. Gene. 2019;707:189-97.

42. Livak KJ, Schmittgen TD. Analysis of relative gene expression data using realtime quantitative $P C R$ and the $2{ }_{-}^{\Delta \Delta} C T$, method. Methods. 2001;25:402-8.

43. Larkin MA, Blackshields G, Brown NP, Chenna R, McGettigan PA, McWilliam $H$, et al. Clustal W and Clustal X version 2.0. Bioinformatics. 2007;23:2947-8.

44. Tamura K, Stecher G, Peterson D, Filipski A, Kumar S. MEGA6: molecular evolutionary genetics analysis version 6.0. Mol Biol Evol. 2013;2013:2725-9.

45. Yao WJ, Wang SJ, Zhou BR, Jiang TB. Transgenic poplar overexpressing the endogenous transcription factor ERF76 gene improves salinity tolerance. Tree Physiol. 2016;36:896-908.

46. Sukjun J, Seunghyun K, Illmin C. Comparison of lignin, cellulose, and hemicellulose contents for biofuels utilization among 4 types of lignocellulosic crops. Biomass Bioenergy. 2015;83:322-7.

47. Liljegren S. Phloroglucinol stain for lignin. Cold Spring Harb Protoc. 2010; 5(1):pdb.prot4954.

\section{Publisher's Note}

Springer Nature remains neutral with regard to jurisdictional claims in published maps and institutional affiliations.

Ready to submit your research? Choose BMC and benefit from:

- fast, convenient online submission

- thorough peer review by experienced researchers in your field

- rapid publication on acceptance

- support for research data, including large and complex data types

- gold Open Access which fosters wider collaboration and increased citations

- maximum visibility for your research: over $100 \mathrm{M}$ website views per year

At $\mathrm{BMC}$, research is always in progress.

Learn more biomedcentral.com/submissions 